Société d'histoire de la révolution de 1848 et des

révolutions du XIXe siècle

$50 \mid 2015$

Sociétés et forces de sécurité au $\mathrm{XIX} \mathrm{X}^{\mathrm{e}}$ siècle

\title{
Güneş IŞIKSEL et Emmanuel SZUREK [dir.], Turcs et Français. Une histoire culturelle, 1860-1960
}

Rennes, Presses universitaires de Rennes, 2014, 387 p. ISBN :

978-2-7535-3349-3. 22 euros.

\section{Cosima Flateau}

\section{OpenEdition}

Journals

Édition électronique

URL : http://journals.openedition.org/rh19/4877

DOI : $10.4000 /$ rh 19.4877

ISSN : $1777-5329$

Éditeur

La Société de 1848

Édition imprimée

Date de publication : 1 juillet 2015

Pagination : 233-235

ISSN : 1265-1354

Référence électronique

Cosima Flateau, « Güneș ISSIKSEL et Emmanuel SZUREK [dir.], Turcs et Français. Une histoire culturelle, 1860-1960», Revue d'histoire du XIXe siècle [En ligne], 50 | 2015, mis en ligne le 01 juillet 2015, consulté le 22 septembre 2020. URL : http://journals.openedition.org/rh19/4877 ; DOI : https://doi.org/ $10.4000 /$ rh 19.4877

Ce document a été généré automatiquement le 22 septembre 2020

Tous droits réservés 


\section{Güneş IşIKSEL et Emmanuel SZUREK [dir.], Turcs et Français. Une histoire culturelle, 1860-1960}

Rennes, Presses universitaires de Rennes, 2014, 387 p. ISBN :

978-2-7535-3349-3. 22 euros.

\section{Cosima Flateau}

\section{RÉFÉRENCE}

Güneş IşIKSEL et Emmanuel SZUREK [dir.], Turcs et Français. Une histoire culturelle, 1860-1960, Rennes, Presses universitaires de Rennes, 2014, 387 p. ISBN :

978-2-7535-3349-3. 22 euros.

1 Issu d'un colloque international qui s'est tenu en mars 2010 à l'École normale supérieure, ce volume préfacé par François Georgeon recueille les contributions d'historiens sur les relations entre Français et Turcs sur un siècle (1860-1960), mettant l'accent davantage sur le $\mathrm{XX}^{\mathrm{e}}$ que sur le XIX ${ }^{\mathrm{e}}$ siècle. L'ouvrage s'ancre dans une historiographie culturelle de la Turquie déjà riche dans les domaines de l'histoire du livre et de la presse, de l'enseignement et des missions, des sciences et des techniques. Cette histoire s'intéresse aux circulations d'objets et de savoirs et surtout aux acteurs, et privilégie souvent des sources françaises plus qu'ottomanes puis turques.

2 Emmanuel Szurek propose d'abord de se démarquer de l'imagerie sentimentale associée à l'amitié franco-turque, établie depuis François ${ }^{\text {er }}$ et Soliman le Magnifique, réaffirmée par la "vocation » française de défense des chrétiens d'Orient, et réveillée dans le cadre d'une histoire culturelle de la Méditerranée en plein renouvellement. Pour établir cette histoire à la fois sociale et transnationale de l'interaction culturelle, il s'agit d'aborder davantage les échanges culturels par les acteurs que par les marchandises. De la guerre de Crimée, qui marque une accélération des échanges franco-turcs, à la Guerre froide, qui voit la montée en puissance des normes anglo- 
américaines, des relations étroites se sont tissées entre France et Turquie. Dans ce siècle d'interactions culturelles intenses, la césure de la Grande Guerre fait passer d'un monde fluide, dans un cadre impérial, à un cadre national, où le territoire et la conscience nationale se structurent.

3 La première partie de l'ouvrage s'attache à l'étude de l'exportation des formes et des vecteurs de la pensée française, à leur réception et à leur réinterprétation dans le cadre ottoman. Au fondement des échanges culturels franco-turcs se trouve la langue française, indispensable au commerce et marque de distinction sociale. Claire Fredj s'intéresse à l'usage du français au sein d'un groupe professionnel, celui des médecins, entre 1839 et 1914. La guerre de Crimée marque une montée en puissance de l'influence française dans le domaine médical, grâce à la diffusion par les médecins militaires des normes pratiques et intellectuelles de la modernité. Cette influence, consolidée par des institutions et des journaux, est ensuite remise en cause par la naissance d'une formation plus "nationale ", incarnée par l'École de médecine militaire ottomane. Les deux contributions suivantes mettent en évidence les modalités d'introduction et d'appropriation du positivisme et du bergsonisme, deux courants philosophiques très en vogue sur la scène intellectuelle ottomane. Enes Kabakc1 montre comment le positivisme est interprété en Turquie par quatre générations intellectuelles successives entre 1860 et 1950 et Dilek Sarmis évoque la revue Dergâh (La Porte), née pendant la guerre d'indépendance turque (1919-1922), qui voit dans le spiritualisme de Bergson une alternative au positivisme érigé en modèle par le Comité Union et Progrès.

4 La deuxième partie de l'ouvrage se penche sur deux catégories d'acteurs: les archéologues et les militaires. Nicole Chevalier met en lumière les enjeux politiques de l'expansion archéologique, perçue entre 1912 et 1939 comme l'expression du génie français, notamment contre l'Allemagne. Le souci des intérêts nationaux se manifeste par l'organisation rationalisée des fouilles à la veille de la Grande Guerre, puis dans le sillage des campagnes militaires du début des années 1920. Les deux contributions suivantes étudient les échanges franco-turcs par l'intermédiaire des militaires, dans un contexte de rivalité franco-allemande. Loubna Lamrhari étudie le point de vue des militaires français face à l'influence militaire allemande sur l'armée ottomane, de l'arrivée de la mission von der Goltz (1883), jusqu'à l'avènement du régime nazi (1933). Le regard des deux générations d'observateurs français se construit en réaction à deux événements clés, le traumatisme de la défaite de 1870 et la fierté de la victoire de 1918. Franziska Heimburger concentre son propos sur le temps court de la Première Guerre mondiale, au cours de laquelle la recherche de compétences linguistiques en turc donne aux interprètes militaires une place centrale dans l'armée d'Orient. C'est enfin encore la concurrence allemande qui engendre au début des années 1930 la création de l'Institut français d'Istanbul, étudiée par Jacques Thobie.

5 La troisième partie s'inscrit dans le contexte de la Turquie née de la révolution kémaliste, qui s'impose sur la scène internationale en donnant une impression de modernité. Olivier Decottignies étudie la figure de Paul Gentizon, correspondant du Temps à Istanbul de 1922 à 1928, défenseur de l'idée d'un «Orient en marche », dont la Turquie serait l'avant-garde éclairée. Timour Muhidine témoigne de la fascination exercée par la forte personnalité de Mustafa Kemal et par ses réalisations politiques, sociales et architecturales sur quelques femmes journalistes, romancières ou touristes voyageant en Anatolie. Les deux articles suivants retracent le parcours de deux experts français dans la Turquie née du kémalisme. Jean-François Pérouse réévalue la figure 
d'Henri Prost, architecte au service de la municipalité d'Istanbul entre 1937 et la fin des années 1950, dont l'œuvre de réagencement de la ville fait toujours débat en Turquie. Pierre Pinon s'attache à la trajectoire d'Albert Gabriel, directeur de l'Institut français d'archéologie d'Istanbul à partir de 1930, qui s'engage en faveur de la promotion de l'art turc.

6 La quatrième partie est consacrée à l'institution universitaire, dans un contexte d'internationalisation et d'autonomisation du champ scientifique turc. Les mobilités universitaires sont examinées par Nicolas Ginsburger, qui aborde le rôle des scientifiques comme vecteurs de la puissance allemande et française. La "période turque » (1926-1940) de Georges Dumézil, retracée par Alexandre Toumarkine, insiste plutôt sur la marginalisation que peut entraîner un séjour en Turquie et les difficultés d'insertion dans les réseaux locaux. Guillaume Tronchet complète la description de ces rencontres "ratées", en évoquant les désillusions réciproques dans les échanges universitaires des années 1930.

7 La cinquième et dernière partie est consacrée à la naissance d'une discipline, la turcologie, et à sa différenciation à l'égard du métier d'orientaliste, pratiqué en premier lieu par les drogmans, étudiés par Gilles Veinstein. Ces derniers, bénéficiant d'une véritable formation depuis la création de l'École des jeunes de langues par Colbert (1669), puis de l'École des langues vivantes orientales (après 1880) sont le premier vivier des futurs turcologues, même si une véritable rupture épistémologique s'opère avec la naissance de cette discipline. La contribution de Guy Basset fait un gros plan sociologique sur la famille et les réseaux familiaux et universitaires de René Basset, doyen de la faculté d'Alger et codirecteur de l'Encyclopédie de l'Islam. Emmanuel Szurek aborde l'émergence de la turcologie comme discipline à part entière et Güneş Işiksel analyse la fin de "l'âge d'or " de l'échange culturel franco-turc, à travers l'élaboration du premier Grundriss de turcologie dans les années 1950. Edhem Eldem conclut l'ouvrage par une sorte d'hommage à l'ouvrage publié en 1962 par Robert Mantran, Istanbul dans la seconde moitié du XVIIIe siècle qui, par le caractère inédit et l'ampleur des sources utilisées, a fait date dans le champ des études turques.

Les contributions de cet ouvrage s'appuient sur une méthodologie renouvelée de l'histoire culturelle, comme l'histoire des réseaux ou celle des transferts, qui permettent de repenser les enjeux liés à la transmission et à l'appropriation des savoirs. Le souci de faire varier les échelles permet de montrer, au-delà de l'image d'une relation privilégiée entre la France et la Turquie et des aléas des relations internationales, que cette histoire des relations culturelles a pu aussi être marquée à l'échelle individuelle par des déceptions ou des incompréhensions. Les relations francoturques sont, au fur et à mesure de la période, de plus en plus organisées et prises en charge par les États, dans le cadre d'une diplomatie culturelle centralisée. On aurait peut-être aimé que ces relations culturelles soient parfois moins examinées à partir des centres politiques (Istanbul puis Ankara), laissant davantage de place aux provinces, ainsi qu'aux échanges et aux acteurs moins institutionnels. 